\title{
Crenças de indivíduos com hipertensão arterial sistêmica relacionadas ao tratamento medicamentoso
}

\author{
Beliefs of individuals with systemic arterial hypertension related to drug treatment
}

Taciana da Costa Farias Almeida ${ }^{1}$, Mailson Marques de Sousa ${ }^{1}$, Marina Saraiva de Araújo Pessoa ${ }^{2}$, Larissa dos Santos Sousa ${ }^{2}$, Bernadete de Lourdes André Gouveia ${ }^{1}$, Simone Helena dos Santos Oliveira ${ }^{1}$

Objetivo: analisar as crenças comportamentais, normativas e de controle relacionadas ao tratamento medicamentoso para hipertensão arterial. Métodos: estudo transversal, fundamentado na Teoria do Comportamento Planejado, realizado com 28 pessoas em uso contínuo de anti-hipertensivos, em acompanhamento ambulatorial. Utilizou-se de formulário com seis perguntas abertas, aplicado por entrevistas estruturadas e individuais, gravadas em áudio e submetidas à análise de conteúdo. Resultados: entre as crenças comportamentais, o controle da pressão arterial destacou-se como principal vantagem, e os sintomas desagradáveis sobressaíram como desvantagem. Com relação às crenças normativas, os referentes sociais positivos mais citados foram os filhos. Quanto às crenças de controle, adquirir gratuitamente e esquecer do horário de tomá-los foram apontados como principais facilidade e dificuldade, respectivamente. Conclusão: a análise das crenças permitiu a elucidação daquelas que podem influenciar positiva ou negativamente o comportamento "tomar os comprimidos prescritos para o controle da hipertensão", alvo de diferentes intervenções em serviços de saúde.

Descritores: Hipertensão; Cooperação do Paciente; Comportamento; Teoria Social; Enfermagem.

Objective: to analyze behavioral, normative and control beliefs related to drug treatment for hypertension. Methods: cross-sectional study, based on the Planned Behavior Theory, conducted with 28 people on continuous use of antihypertensive drugs, in outpatient follow-up. A form with six open questions was used, applied by structured and individual interviews, audio recorded and submitted to content analysis. Results: among the behavioral beliefs, blood pressure control stood out as the main advantage, and unpleasant symptoms stood out as a disadvantage. Regarding normative beliefs, the most cited positive social references were the children. As for control beliefs, acquiring for free and forgetting the time to take them were pointed as the main ease and difficulty, respectively. Conclusion: the analysis of beliefs allowed the elucidation of those that may positively or negatively influence the behavior of "taking the prescribed pills for the control of hypertension", target of different interventions in health services.

Descriptors: Hypertension; Patient Compliance; Behavior; Social Theory; Nursing.

\footnotetext{
${ }^{1}$ Universidade Federal da Paraíba. João Pessoa, PB, Brasil.

${ }^{2}$ Universidade Federal de Campina Grande. Campina Grande, PB, Brasil. gmail.com
} 


\section{Introdução}

A hipertensão arterial sistêmica é uma afecção cardiovascular e importante fator de risco para esta. A baixa adesão ao tratamento da hipertensão pode favorecer o aumento das taxas de morbidade e mortalidade por essa doença. Entre os fatores relacionados a esta baixa adesão, o uso de medicamentos sem prescrição médica ou erros relacionados à dose e horário são descritos como elementos prejudiciais para controle dos níveis pressóricos, o que torna o controle da morbimortalidade pela doença desafio para o sistema público de saúde ${ }^{(1-2)}$.

A não adesão ao tratamento, comportamento estudado no âmbito da saúde, pode estar associada à manifestação de crenças irracionais e racionais, fatores cognitivos, carência de informação sobre os riscos de não adesão ou habilidades comportamentais e de enfrentamento diminuídas ${ }^{(3-4)}$.

Nesse contexto, no estudo de comportamentos em saúde, a Teoria do Comportamento Planejado vem sendo empregada para compreender, explicar e predizer fenômenos em contextos específicos. De maneira geral, considera que o comportamento é estabelecido pela intenção no agir e percepção de controle ${ }^{(5)}$.

A intenção, um dos precedentes imediatos do comportamento, é determinada por três variáveis: a atitude, formada pelas crenças comportamentais, que refletem as vantagens e desvantagens de realizar o comportamento; normas subjetivas, constituídas pelas crenças normativas, referentes sociais que aprovam ou desaprovam a efetivação do comportamento; e o controle comportamental percebido, composto por crenças de controle, que identificam facilidades e dificuldades para realizar o comportamento ${ }^{(5)}$.

Portanto, para predizer a intenção comportamental, a partir dos conceitos de atitude, norma subjetiva e controle comportamental percebido, torna-se primordial a elucidação das crenças salientes em relação ao fenômeno investigado.

Crenças salientes são as mais frequentes, isto é, mencionadas mais vezes e originadas a partir de uni- verso e assunto comum em relação ao comportamento definido. Neste escopo, o comportamento de interesse para o estudo foi definido como: tomar os comprimidos prescritos para controle da hipertensão arterial, nos próximos 30 dias, que abrange os elementos de alvo, ação, contexto e tempo, conforme pressupostos do referencial teórico adotado ${ }^{(5-6)}$.

Ao averiguar o estado da arte, foram localizados estudos prévios no cenário brasileiro, relacionados a crenças de pacientes sobre hipertensão arterial sistêmica, de maneira global ao tratamento ${ }^{(4,7-8)}$. Contudo, estudos voltados à identificação das crenças de indivíduos com hipertensão acerca do comportamento de tomar os comprimidos prescritos para controlá-la, à luz do referencial teórico adotado neste estudo, são incipientes. Em concordância com a complexidade do comportamento de aderir ao tratamento medicamentoso, justifica-se a relevância deste estudo.

Ademais, considera-se salutar que profissionais de saúde que assistem indivíduos com hipertensão arterial sistêmica conheçam e abordem fatores inerentes às crenças ao tratamento, em especial quanto ao comportamento de tomada dos medicamentos anti-hipertensivos, uma vez que a não adesão inviabiliza o gerenciamento eficaz da doença.

Diante do exposto, objetivou-se analisar as crenças comportamentais, normativas e de controle relacionadas ao tratamento medicamentoso para hipertensão arterial.

\section{Métodos}

Estudo descritivo, transversal, norteado pela Teoria do Comportamento Planejado, realizado em ambulatório de cardiologia de hospital universitário, em município do interior do Estado da Paraíba, Brasil.

De acordo com a Teoria do Comportamento Planejado, não há exigência em relação ao número mínimo de respondentes para elucidar as crenças salientes sobre o comportamento a ser investigado. Contudo, o critério de saturação dos dados ${ }^{(6)}$ deve ser respeitado, ou seja, quando novas informações não fo- 
rem mais obtidas, redundância das respostas, deve-se interromper a coleta dos dados.

Assim, nesta pesquisa, a saturação das respostas foi alcançada com declaração de 28 respondentes. A amostra selecionada por conveniência constou de indivíduos que atenderam aos critérios de inclusão: idade $\geq$ a 18 anos, com diagnóstico de hipertensão arterial sistêmica, em uso contínuo de anti-hipertensivos orais há pelo menos seis meses e em acompanhamento ambulatorial. Não foram inclusas pessoas com hipertensão arterial sistêmica que tinham a administração dos comprimidos gerenciada por cuidador e que apresentavam barreiras cognitivas registradas em prontuário. Não houve recusas em participar do estudo, sendo inseridos todos os participantes convidados, consecutivamente.

A obtenção dos dados ocorreu de dezembro de 2017 a janeiro de 2018, com utilização de dois instrumentos, elaborados pelos pesquisadores, avaliados por membros do grupo de estudo e doenças crônicas. Os instrumentos foram submetidos à avaliação e pré-teste $^{(6)}$ por cinco pessoas com hipertensão arterial sistêmica para avaliar a compreensão e realizar as adequações necessárias. 0 primeiro direcionado à caracterização sociodemográfica e clínica, continha as variáveis idade, sexo, com quem reside, escolaridade e renda familiar, estágio de classificação da hipertensão arterial sistêmica, de acordo com as VII Diretrizes Brasileiras de Hipertensão ${ }^{(9)}$, tempo de diagnóstico e medicações anti-hipertensivas em uso.

O segundo, um formulário com seis questões abertas, duas para cada tipo de crença (positiva/negativa), elaboradas segundo os pressupostos da Teoria do Comportamento Planejado ${ }^{(5)}$, aplicado mediante entrevista estruturada individual, em ambiente privativo no local da pesquisa, gravadas em aparelho smartphone, no modo offline. 0 procedimento de coleta de dados durou em média dez minutos.

Para o levantamento das crenças comportamentais, foram elaboradas as perguntas: na sua opinião, quais as vantagens/desvantagens de tomar os comprimidos prescritos para o controle da hipertensão arterial, nos próximos 30 dias? Para as crenças normativas, questionou-se: na sua opinião, quais as pessoas importantes para você que acham que você deve/não deve tomar os comprimidos prescritos para o controle da hipertensão arterial, nos próximos 30 dias? Por fim, para as crenças de controle, perguntou-se: na sua opinião, quais as facilidades/dificuldades que você encontra para tomar os comprimidos prescritos para o controle da hipertensão arterial, nos próximos 30 dias?

No que tange às crenças frente ao comportamento tomar os anti-hipertensivos orais conforme prescrito, nos próximos 30 dias, as entrevistas foram transcritas na íntegra pelo pesquisador principal, no software Microsoft Office Word 2013, versão $15.0^{\circledR}$, e, posteriormente, submetidas à análise descritiva e de conteúdo, com base nas recomendações da teoria ${ }^{(5-6)}$. Para assegurar o anonimato, os discursos foram identificados com a letra “h", em referência à palavra hipertenso, seguido do número, ao final das falas, conforme a sequência de realização das entrevistas.

A análise dos dados seguiu as etapas: exploração do material, no intuito de identificar os constructos da teoria estudada; agrupamento das respostas em cada constructo, de acordo com as falas dos participantes; contabilização da frequência das emissões, sendo destacadas as crenças modais salientes ou mais frequentes.

Para identificar quais crenças seriam incluídas, utilizaram-se dos critérios propostos pela Teoria do Comportamento Planejado, que classifica como crenças modais salientes as que excedem determinada frequência e totalizam pelo menos $75,0 \%$ das crenças emitidas ${ }^{(5-6)}$. Para este estudo, o agrupamento das crenças ocorreu a partir da contabilização das emissões, sendo selecionadas aquelas emitidas no mínimo cinco vezes e que, no seu conjunto, somaram $75,0 \%$ ou mais das crenças.

O estudo respeitou as normas nacionais e internacionais de pesquisas envolvendo seres humanos. Foi aprovado pelo Comitê de Ética em Pesquisa de Hospital Universitário do interior do Estado da Paraíba, conforme parecer no 2.446.615/2017. 


\section{Resultados}

Participaram 28 pessoas com hipertensão arterial sistêmica, com predomínio do sexo feminino 23 $(82,1 \%)$, média de idade de 55 anos e escolaridade de oito anos de estudo; $24(85,7 \%)$ residiam com familiares e renda familiar mensal que variava de um a três salários mínimos.

O tempo de diagnóstico variou de seis meses a 21 anos, considerando o critério adotado pela VII Diretriz Brasileira de Hipertensão, os valores pressóricos aferidos classificaram os participantes em Pressão Arterial Ótima, quatro (14,3\%); Normal, três $(10,7 \%)$; Limítrofe, quatro (14,3\%); hipertensão Estágio I, dois (7,1\%); hipertensão Estágio II, dois $(7,1 \%)$; e hipertensão Sistólica Isolada, 13 (46,4\%).

Quanto à utilização de fármacos para controlar a hipertensão arterial sistêmica, destacaram-se as classes de anti-hipertensivos: antagonistas do receptor da angiotensina II, 20(71,9\%); diuréticos, 17 (60,7\%); bloqueadores de canais de cálcio, 10 $(35,7 \%)$; beta bloqueador, nove $(32,1 \%)$; inibidores da enzima conversora de angiotensina II, cinco $(17,9 \%)$; inibidores adrenérgicos, um $(3,6 \%)$; e vasodilatadores, um $(3,6 \%)$.

No intuito de explorar as crenças emitidas, as categorias de análise foram organizadas de acordo com os constructos pré-fixados da Teoria do Comportamento Planejado (crenças comportamentais; crenças normativas e crenças de controle) e frequências de emissões, conforme evidenciados nas falas que seguem.

\section{Crenças Comportamentais}

Dentre as vantagens $(\mathrm{n}=46)$, destacou-se controlar a pressão arterial, 17 (37,0\%); evitar complicações e morte, nove (19,6\%); sentir-me bem, oito $(17,4 \%)$, evitar sintomas da doença (dor de cabeça, dor nas pernas e enjoo), sete (15,2\%); e proporcionar segurança e tranquilidade em relação a minha saúde, cinco $(10,8 \%)$.
As falas revelaram que a partir das crenças, emergiram a importância da aderência ao comportamento pesquisado. Eu acho que pelo menos melhora e controla a minha pressão, e é um fator para não ter outra doença a mais, entendeu?...no caso, um infarto ou algo parecido e morrer (h5). Assim, eu me sinto mais segura e tranquila em relação a minha saúde... (h8). Após começar a tomar o remédio, o captopril, no caso, eu diminuí 50,0\% das dores de cabeça que eu sentia, eu sempre passava mal... ânsia (enjoo), vários sintomas, mas após tomar, regulou muito a minha pressão arterial e o meu bem-estar (h19).

Com relação às desvantagens $(n=37)$ apontadas, evidenciaram-se os efeitos adversos (mal-estar, agonia, dor no estômago, diminuição da libido, irritabilidade, ir várias vezes ao banheiro e tosse), 11 $(29,7 \%)$, e também o tratamento ser para o resto da vida, o que causa dependência, cinco $(13,5 \%)$, como pode ser evidenciado nas falas. É, muitas vezes, diminui a libido, entendeu?... irritabilidade..., e aquela, tem que ter aquele horário definido para o resto da vida... (h1). Assim, o mal-estar que eu sinto no estômago ...Eu sinto um mal-estar muito grande no estômago, por sinal, até já falei para a doutora. Eu sinto uma agonia, entendeu? (h2). Às vezes, eu sinto agonia... não é toda vez não, é às vezes (h3). Porque se esquecer ou não tomar (dependente), ela (pressão arterial) vai lá para cima e a gente começa a passar mal (h7). Eu acho ruim. Todos os dias, tem que ser essa rotina. É uma total dependência (h14). O captopril dá, às vezes, bastante tosse, que é um sintoma que várias pessoas dizem que ele faz mesmo ...e isso incomoda (h19). É porque a gente vai muito para o banheiro (h25).

E $12(34,2 \%)$ crenças foram emitidas em relação a nenhuma desvantagem pelo uso dos anti-hipertensivos.

\section{Crenças Normativas}

Em analogia aos referentes positivos ( $\mathrm{n}=52)$, os participantes destacaram filhos, 17 (37,2\%); esposo/ esposa, oito (17,0\%); médicos, sete (14,9\%); e família, sete $(14,9 \%)$, como os referentes sociais que aprovavam a tomada de anti-hipertensivo, conforme evidenciado nas falas. São meus filhos (risos). É, eles ficam pedindo, mãe não esqueça...(h3). Tem meu marido, é que ele tem muito cuidado, sabe (risos)? (h11). A minha família, somente (h11). Os médicos, 
sempre recebo uma reclamação quando a pressão está alta (h13).

Observou-se, nas falas dos participantes, que $27(93,0 \%)$ referiram não haver referente social negativo, ou seja, que desaprovavam a tomada do anti-hipertensivo para tratamento da hipertensão. Ninguém nunca falou (h1). Não. Até agora ninguém nunca disse isso não (h4). Identificou-se que apenas um sujeito, 0,7\%, relatou que o vizinho o incentivava, negativamente, a não realizar o tratamento.

\section{Crenças de Controle}

Quanto aos fatores que facilitavam $(n=44)$ a tomada de anti-hipertensivos, apontaram: adquirir os comprimidos gratuitamente, 14 (31,8\%); ato de tomar os comprimidos (colocar na boca com água e engolir), 11 (25,0\%); e baixo custo dos medicamentos, $10(22,8 \%)$. As falas revelaram estas crenças de controle. Já tenho o comprimido pronto, é só ingerir com água (h1). Eu tenho que comprar, não existe grátis. Mas, não fica muito caro não, é acessível. Dá para comprar, graças a Deus (h2). É fácil tomar, pois a gente recebe grátis. Recebe no posto... (h7).

A respeito daqueles que dificultavam $(n=32)$ a tomada dos comprimidos, foram reportados o esquecimento do horário, oito (25,0\%), e a necessidade de comprar, cinco $(15,6 \%)$. Horário, eu tenho assim os horários estabelecidos, às vezes, quando eu durmo um pouco mais, eu perco o horário... mas, ela (a médica) falou agora que eu posso, assim que automaticamente comer, ingeri-lo, eu não sabia (h1). A dificuldade, às vezes, é comprar, quando falta... sempre falta (h24).

E $15(46,8 \%)$ crenças foram emitidas em relação a nenhuma dificuldade para fazer uso dos anti-hipertensivos.

\section{Discussão}

Como limitação para o estudo, destaca-se que os resultados são restritos à amostra coletada. Portanto, os achados obtidos não podem ser estendidos para todos os indivíduos com hipertensão arterial sistêmica, não sendo possível a generalização das crenças quanto ao objetivo proposto.
Para prática de Enfermagem, as crenças elucidadas servirão como subsídio para estruturação de escala psicométrica para avaliar os fatores determinantes da intenção comportamental em realizar o comportamento estudado. Os resultados obtidos contribuirão para elaboração de estratégias motivacionais, a partir do contexto regional, com intuito de influenciar positivamente a tomada de anti-hipertensivos, beneficiando a adesão terapêutica medicamentosa.

Compreende-se que a amostra investigada conhecia as vantagens de aderir ao tratamento prescrito, ainda que apresentassem níveis pressóricos não controlados, uma vez que pequena proporção dos indivíduos apresentava valores pressóricos classificados como ótimos ou normais. Salienta-se que o controle da hipertensão arterial sistêmica se faz por medidas farmacológicas e não farmacológicas ${ }^{(9)}$, não sendo possível inferir que o descontrole dos níveis pressóricos encontrados possua relação direta com a tomada dos anti-hipertensivos de forma incorreta.

Detectou-se como crenças comportamentais positivas a preocupação das pessoas com hipertensão arterial sistêmica em manter o controle da pressão arterial, evitar complicações e morte, sentir-se bem, evitar sintomas e segurança em relação à saúde. Estes achados são condizentes e relacionados às consequências do efeito do fármaco em uso, resultados que corroboram com estudo realizado com afro-americanos nos Estados Unidos ${ }^{(10)}$.

Estudos realizados na China, Chile e Estados Unidos $^{(2-3,11)}$ também identificaram, entre as crenças comportamentais negativas, desvantagens em relação aos efeitos adversos dos fármacos e a cronicidade do tratamento, postergando-o por longo período da vida, o que pode causar dependência. Torna-se importante conhecer estas crenças, no intuito de minimizar sua valorização, ponderando que as vantagens devem sobrepor as desvantagens do tratamento.

Entre os efeitos adversos percebidos ou reais induzidos pelos medicamentos, foram elucidados como mal-estar, agonia, dor no estômago, diminuição 
da libido, irritabilidade, ir várias vezes ao banheiro ou tosse, também identificados em outros estudos prévios $^{(11-12)}$. Importante sinalizar que muitos desses efeitos adversos podem não estar relacionados diretamente ao uso dos fármacos anti-hipertensivos, mas ao descontrole dos níveis pressóricos ${ }^{(8)}$, que pode estar relacionado ao controle ineficaz da doença.

Torna-se importante que o profissional de saúde, envolvido com a assistência ao indivíduo com hipertensão arterial sistêmica, conheça as medicações em uso, assim como os efeitos adversos dos fármacos e os sintomas de descontrole dos níveis pressóricos, a fim de orientar as pessoas com hipertensão em relação aos possíveis efeitos adversos relatados, que podem não estar relacionadas diretamente à ingestão dos anti-hipertensivos ${ }^{(9)}$.

A saber, a poliúria é um dos efeitos adversos dos medicamentos da classe dos diuréticos, medicamento amplamente utilizado pelos investigados. Os fármacos desta classe podem ainda provocar fraqueza, câimbras, hipovolemia e disfunção erétil ${ }^{(9)}$. 0 conhecimento da ação destes medicamentos possibilita ao usuário melhor manejo de tomada, evitando-se tomá-lo em horários que dificultem a ida ao banheiro em maior frequência, queixa relatada pelos respondentes, facilitando uso e aderência ao tratamento.

A despeito da alteração da libido, efeito adverso que pode estar relacionado à disfunção erétil, ocasionada pelo uso das classes de medicamentos diuréticos, alfa-agonistas de ação central, beta bloqueadores ${ }^{(9)}$, é outro ponto importante descrito em outros estu$\operatorname{dos}^{(11-12)}$, cujos indivíduos do sexo masculino referiram como o uso dos medicamentos afeta o funcionamento sexual, resultando em desafios subsequentes para relações afetivas e visões de masculinidade.

A tosse seca, também, foi relatada pelos respondentes como efeito desagradável e que incomoda a socialização, dado que se destaca na literatura como principal efeito colateral dos anti-hipertensivos da classe dos inibidores da enzima conversora da angiotensina e que acomete 5,0 a $20,0 \%$ dos pacientes com hipertensão ${ }^{(9)}$.
Portanto, torna-se importante que os membros da equipe interprofissional conheçam as crenças comportamentais negativas em relação à tomada dos medicamentos anti-hipertensivos e orientem os indivíduos com hipertensão arterial sistêmica acerca da terapêutica instituída, assim como possibilidades de alterações na prescrição, quando algum destes efeitos adversos estiver presente, uma vez que, quando a pessoa com hipertensão supervaloriza as crenças negativas às positivas, há maior condicionamento ao abandono do tratamento ou baixa adesão, o que incorre em desfechos desfavoráveis da doença ${ }^{(1)}$.

Ser dependente do tratamento, pois a não realização deste pode acarretar sintomas e complicações da doença, também é percebido como desvantagem do tratamento, achado também identificado em estudos realizados em outros países ${ }^{(3,11-12)}$. Os resultados apontam para necessidade de programar estratégias individualizadas e/ou em grupos, em que tecnologias de cuidado em saúde possam ser testadas e efetivadas para esta população, no intuito de suprimir esta barreira e impulsionar a adesão aos anti-hipertensivos orais, considerando a importância do tratamento para gestão da saúde e percepção da qualidade de vida.

Em relação às crenças normativas, os referentes sociais positivos, filhos (as) e esposa (o), médico e a família, de modo geral, são considerados como agentes motivacionais para tomada do anti-hipertensivo. Compreende-se que ter uma rede de apoio social que envolva a importância do tratamento é substancial no cuidado e acompanhamento do indivíduo com hipertensão ${ }^{(3)}$, uma vez que o apoio social e o valor atribuído à família ajudam a pessoa com hipertensão a seguir o tratamento prescrito, e devem ser considerados, no momento de elaborar estratégias de ação, para melhorar a adesão ao tratamento anti-hipertensivo ${ }^{(2,11)}$.

Estar por perto da família condiciona resultados positivos em relação à adesão aos anti-hipertensivos, uma vez que a família exerce papel motivador no seguimento da terapêutica. 0 incentivo à adesão pelo médico e a aprovação da família geram sentimento de gratidão nas pessoas com hipertensão arterial sistê- 
mica, uma vez que estas pessoas se configuram como importantes agentes de apoio ao bem-estar e escolhas saudáveis em saúde ${ }^{(3,11)}$.

Investigação nacional que utilizou a Teoria do Comportamento Planejado para identificar as crenças relacionadas à adesão ao tratamento de antidiabético oral evidenciou filhos, esposo, médico e equipe de enfermagem como referentes sociais positivos à adesão medicamentosa $^{(13)}$. Dados que corroboram parcialmente com os achados deste estudo, uma vez que a equipe de enfermagem não foi referida.

Esse fato pode ser explicado, particularmente, ao lócus onde foi desenvolvido o estudo, no qual apenas o profissional médico atuava no acompanhamento clínico de pacientes com hipertensão arterial sistêmica. Deste modo, aponta-se a necessidade de incorporar o enfermeiro na assistência ambulatorial oferecida aos indivíduos com hipertensão arterial sistêmica, uma vez que a consulta de enfermagem é um instrumento de ação que permite o levantamento dos fatores que interferem no processo de adesão ao tratamento anti-hipertensivo, relacionados aos aspectos biopsicossociais, crenças e conhecimentos sobre a doença, os hábitos, o estilo de vida e o tratamento ${ }^{(4)}$.

Ao concordar com essa assertiva, estratégias desenvolvidas na prática clínica do enfermeiro, entre elas: visitas domiciliares ${ }^{(14)}$, contatos telefônicos ${ }^{(15)}$ e envio de mensagens de texto ${ }^{(16)}$ têm demonstrado resultados satisfatórios na adesão ao tratamento medicamentoso e podem constituir-se como ferramenta adjuvante na abordagem terapêutica, a fim de reverter crenças negativas em positivas e impulsionar adesão ao tratamento anti-hipertensivo.

Enfatiza-se a ausência de referentes negativos, ou seja, pessoas que desaprovam o comportamento estudado. A única emissão referida foi relacionada a pessoas externas à família e/ou equipe de saúde, que no caso um vizinho. Assim, torna-se importante reforçar os elos entre as pessoas que são importantes para o indivíduo com hipertensão, especialmente nos momentos de instituição e alterações em terapêuti- cas, uma vez que são pessoas que se preocupam com esta clientela e poderá pontuar positivamente na ação prescrita $^{(1)}$.

Identificou-se, em estudo realizado no Brasil ${ }^{(13)}$, que entre as facilidades emitidas pelos participantes para realizar o tratamento antidiabético estava em adquirir os comprimidos gratuitamente, que coincide com a política de acessibilidade aos fármacos para o tratamento de doenças crônicas, de forma gratuita ou a baixo custo no país. Além desta crença, identificou-se a facilidade para tomá-lo com água, crença não citada em estudo referido.

Políticas públicas, como o Programa Remédio em Casa, facilitam ter o medicamento gratuitamente e acesso pleno às medicações anti-hipertensivas, o que contribui para adesão medicamentosa ${ }^{(17)}$. Esta iniciativa favorece e motiva o seguimento terapêutico, ao observar que a maior parcela de indivíduos hipertensos são usuários do Sistema Único de Saúde, que apresentam baixa escolaridade e renda, o que se torna facilidade, quando este é acessível de forma gratuita ou a baixo custo.

Entre as dificuldades encontradas para tomar os anti-hipertensivos, apontou-se: quando há necessidade de comprar e esquecimento da tomada dos fármacos, fatores relatados também em estudos anteriores $^{(4,10,12)}$. Estes achados refletem a fragilidade na política de dispensação de medicamentos essenciais para manutenção da estabilidade clínica da hipertensão arterial sistemática. Enfatiza-se que a necessidade de comprá-los constitui barreira para adesão terapêutica ${ }^{(4)}$ e, por conseguinte, não adesão ao tratamento prescrito, fator relacionado à baixa renda da maioria dos indivíduos investigados.

Assim como estudos realizados no Brasil e Jamaica ${ }^{(4,12)}$, o esquecimento da tomada dos fármacos se configurou como dificuldade relevante em relação ao tratamento da hipertensão. Apesar ser considerado fator cognitivo inerente à subjetividade de cada indivíduo, quando as pessoas compreendem que este está interferindo negativamente no tratamento, pode- 
-se utilizar de estratégias para melhorá-lo, como pedir ajuda à rede de apoio social (referentes positivos) $(2,11)$ ou utilizar de artefatos para lembrar-se de tomar, como lembretes colocados em locais visíveis, alarmes sonoros, aplicativos para celular, entre outros.

A utilização de estratégias motivadoras, com uso de tecnologias inovadoras, com foco nas necessidades individuais, que promovam apoio social, lembretes guiados por mensagens informativas e incentivadoras relacionadas à importância de tomar os comprimidos, com suporte de smarthphones, devem ser pensadas, construídas e aplicadas a esta população, a fim de incentivar a adesão ao tratamento e enfrentar obstáculos e, consequentemente, melhorar a gestão de saúde dos indivíduos com hipertensão(18).

\section{Conclusão}

As crenças dos indivíduos com hipertensão arterial sistêmica sobre o tratamento medicamentoso que se destacaram foram: controle da pressão arterial, sintomas desagradáveis dos fármacos, filhos como referentes sociais positivos, aquisição gratuita dos medicamentos e esquecimento do horário de tomá-los.

Os achados podem subsidiar o desenvolvimento de estratégias para reverter crenças negativas em positivas, a fim de potencializar a adesão à terapêutica medicamentosa e favorecer o controle da hipertensão arterial, doença crônica, cujo avanço progressivo nos últimos anos tem implicado importantes complicações cardiovasculares e morte.

\section{Colaboradores}

Almeida TCF e Oliveira SHS colaboraram na concepção do projeto, análise e interpretação dos dados e aprovação da versão final a ser publicada. Sousa MM, Pessoa MSA, Sousa LS e Gouveia BLA cooperaram com redação do artigo, revisão crítica relevante do conteúdo intelectual e aprovação da versão final a ser publicada.

\section{Referências}

1. Ma C. An investigation of factors influencing selfcare behaviors in young and middle-aged adults with hypertension based on a health belief model. Heart Lung. 2018; 47(2018):136-41. doi: http:// dx.doi.org/10.1016/j.hrtlng.2017.12.001

2. Yang S, He C, Zhang X, Sun K, Wu S, Sun X, Li Y. Determinants of antihypertensive adherence among patients in Beijing: Application of the health belief model. Patient Educ Couns. 2016; 99:1894-900. doi: http://dx.doi.org/10.1016/j.pec.2016.06.014

3. HerreraPA,MoncadaL, DefeyD.Understandingnonadherence from the inside: hypertensive patients' motivations for adhering and not adhering. Qual Health Res. 2017; 27(7):1023-34. doi: http:// dx.doi.org/10.1177/1049732316652529

4. Pierin AMG, Silva SSBE, Colósimo FC, Toma GA, Serafim TS, Meneghin P. Chronic and asymptomatic diseases influence the control of hypertension treatment in primary care. Rev Esc Enferm USP. 2016; 50(5):764-71. doi: http://dx.doi. org/10.1590/S0080-623420160000600008

5. Ajzen I. The theory of planned behavior. Organ Behav Hum Decis Process. 1991; 50:179-211. doi: https://doi.org/10.1016/0749-5978(91)90020-T

6. Fishbein M, Ajzen I. Predicting and changing behavior: the reasoned action approach. New York: Routledge; 2015.

7. Costa LA, Bresan D, Kawakame PMG, Sales APA. A hipertensão arterial sistêmica na perspectiva de uma comunidade ribeirinha: uma abordagem transcultural. Rev Interd Cult Soc [Internet]. 2017 [citado 2019 Jul 15]; 3:13-30. Disponível e m: http: / / www.periodicoseletronicos. ufma.br/index.php/ricultsociedade/article/ view/6677/4298

8. Silva MGC, Domingos TS, Caramaschi S. Hipertensão arterial e cuidados com a saúde: concepções de homens e mulheres. Psicol Saúde Doenç. 2018; 19(2):435-52. doi: http://dx.doi. org/10.15309/18psd190221

9. Malachias MVB, Souza WKSB, Plavnik FL, Rodrigues CIS, Brandão AA, Neves MFT, et al. 7̣ㅗ Diretriz Brasileira de Hipertensão Arterial. Arq Bras Cardiol. 2016; 107(Supl.3):1-83. doi: http:// dx.doi.org/10.5935/abc.20160152 
10. Bennett J. Beliefs and attitudes about medication adherence in african american men with high blood pressure. Medsurg Nurs [Internet]. 2015 [cited Jul 15, 2019]; 22(3):4-10. Available from: http://www.amsn.org/matters_may13_matters_ newsletter

11. Long E, Ponder M, Bernard S. Knowledge, attitudes, and beliefs related to hypertension and hyperlipidemia self-management among African American men living in the southeastern United States. Patient Educ Couns. 2017; 100(2017):1000-6. doi: http://dx.doi.org/10.1016/j.pec.2016.12.011

12. Wilson TT, Wiliams-Johnson J, Gossel-Williams M, Goldberg EM, Wilks R, Dagupta S, et al. Elevated blood pressure and illness beliefs: a cross-sectional study of emergency department patients in Jamaica. Int J Emerg Med. 2018; 11(30):1-6. doi:http://dx.doi.org/10.1186/s12245-0180187-6

13. Jannuzi FF, Rodrigues RCM, Cornélio ME, São-João TM, Gallani MCBJ. Beliefs related to adherence to oral antidiabetic treatment according to the Theory of Planned Behavior. Rev Latino-Am Enfermagem. 2014; 22(4):529-37. doi: http://dx.doi. org/10.1590/0104-1169.3578.2448

14. Gaziano TA, Bertam M, Tollman SM, Holfman KJ. Hypertension education and adherence in South
Africa: a cost-effectiveness analysis of community health workers. BMC Public Health. 2014; 14(240):1-9. doi: https://doi.org/10.1186/14712458-14-240

15. Raimundo ACN, Pierin AMG. Adherence to anti-hypertensive treatment within a chronic disease management program: a longitudinal, retrospective study. Rev Esc Enferm USP. 2014; 48(5):811-9. doi: http://dx.doi.org/10.1590/ S0080-623420140000500006

16. Bobrow K, Brennam T, Springer D, Levitt NS, Rayner B, Namane M, et al. Efficacy of a text messaging (SMS) based intervention for adults with hypertension: protocol for the StAR (SMS Text-message Adherence suppoRt trial) randomised controlled Trial. BMC Public Health. 2014; 11(14):1-9. doi: http://dx.doi.org/10.1186/1471-2458-14-28

17. Mansour SN, Monteiro CN, Luiz OC. Adherence to medication among hypertensive patients participating in the Medicine at Home Program. Epidemiol Serv Saúde. 2016; 25(3):647-54. doi: http://dx. doi.org/10.5123/S1679-49742016000300021

18. Anderson K, Burford O, Emmerton L. Mobile health apps to facilitate self-care: a qualitative study of user experiences. PLoS One. 2016; 11(5):e0156164. doi: http://dx.doi.org/10.1371/ journal.pone.0156164.eCollection 2016 